\title{
A biphasic system for primary isolation of mycobacteria compared to solid medium and broth culture
}

\author{
S. E. HOFFNER, M. HAILE and G. KÄLLENIUS \\ Department of Bacteriology, The National Bacteriological Laboratory, S-105 21 Stockholm, Sweden
}

\begin{abstract}
Summary. A biphasic culture system, the MB Check, was compared with conventional culture on Löwenstein-Jensen egg (LJ) solid medium and with Bactec broth culture for primary isolation of mycobacteria from clinical samples. A total of 104 mycobacterial isolates was detected from 985 samples examined by the three methods. The most sensitive primary isolation was with LJ culture and MB Check; these methods detected $93 \%$ and $87 \%$ of all positive cultures, respectively. MB Check allowed a somewhat more rapid detection than LJ culture. The presence of atypical mycobacteria was demonstrated most rapidly with the Bactec system.
\end{abstract}

\section{Introduction}

Traditionally, mycobacterial culture is performed on solid medium, with either the egg-based Löwenstein-Jensen (LJ) medium or an agar-based medium such as Middlebrook $7 \mathrm{H} 10$. We and others have shown that solid culture is not optimal for demonstrating atypical mycobacteria such as the Mycobacterium avium complex ${ }^{1}$ and $M$. malmoense. ${ }^{2}$ Broth culture is a potentially important component, that has been called the "modern guinea-pig for isolation of mycobacteria." 3 Recently it was suggested by the European Society for Mycobacteriology that three different types of media, including one broth, should be used for mycobacterial culture. ${ }^{4}$ Since the introduction of the ${ }^{14} \mathrm{C}$-labelled Middlebrook $7 \mathrm{H} 12$ medium $^{5}$ and Bactec radiometry (Becton Dickinson, MD, USA), broth culture has been generally adopted as a diagnostic tool in clinical mycobacteriology.

In this study the MB Check system (F. Hoffman-La Roche, Basel, Switzerland), a biphasic system which combines solid and broth culture for the primary isolation of mycobacteria, ${ }^{6}$ was compared with conventional culture on $\mathrm{LJ}$ medium and with Bactec culture (selected specimens, mainly from extra-pulmonary sources).

\section{Materials and methods}

\section{Specimens}

A total of 985 consecutive routine samples sent to the National Bacteriological Laboratory for mycobacterial culture between October 1990 and February
1991 was included in the study. Most samples were pulmonary in origin (sputum 335, bronchial secretions 159, gastric washings 203). Extra-pulmonary specimens, such as urine, lymph node, bone marrow and cerebrospinal fluid $(76,16,11$ and 8 samples, respectively) were also included. Blood, for which the MB-Check system is not recommended, was excluded.

Samples suspected to be contaminated with nonmycobacterial micro-organisms were decontaminated by the sodium lauryl sulphate method. ${ }^{4}$

\section{The MB Check mycobacterial culture system}

The biphasic culture system (MB Check system) consists of a bottle containing a modified Middlebrook 7H9 broth supplemented with OADC (oleic acid, albumin, dextrose and catalase), glycerol, POES (polyoxyethylene-40-stearate) and PANTA (polymyxin B, amphotericin B, nalidixic acid, trimethoprim and azlocillin), communicating with a plastic cylinder containing a plastic dip-slide supporting three different agar slides (Middlebrook 7H11 agar with and without NAP, $\rho$-nitro- $\alpha$-acetylamino- $\beta$-hydroxypropiophenone, and chocolate agar).

A $0.5-\mathrm{ml}$ volume of the homogenised specimen was inoculated into the broth. The bottles were incubated at $37^{\circ} \mathrm{C}$ and the agar surfaces were flushed once a week with the broth. The cultures were checked for growth twice during the first week, and thereafter once weekly for a further 7 weeks. When growth was visible, acidfast microscopy was done to verify the presence of acid-fast rods.

\section{Solid culture on LJ egg medium}

A $0.5-\mathrm{ml}$ volume of the homogenised specimen was inoculated into one standard $\mathrm{LJ}$ tube and in one with 
pyruvate $0.6 \%$. The tubes were incubated at $37^{\circ} \mathrm{C}$ for 7 weeks (the first week in a $\mathrm{CO}_{2} 5 \%$ atmosphere) and checked once a week for mycobacterial growth. Presence of mycobacteria was verified by acid-fast microscopy.

\section{Bactec broth culture}

The Bactec system was used as described earlier ${ }^{1}$ for selected, mainly extra-pulmonary, samples with a low load of contaminating growth. No antimicrobial agents were added to the culture medium. Of the original 985 specimens, 386 were cultured with the Bactec system, and were compared in all three culture systems.

The isolates were identified by conventional biochemical methods, ${ }^{7}$ and in some cases also with AccuProbe (GenProbe, San Diego, CA, USA).

\section{Results}

Mycobacteria were isolated from $104(10.6 \%)$ of the 985 samples. Organisms of the $M$. tuberculosis complex, including three strains of $M$. bovis, were the most common isolates (83). $M$ avium complex (MAC) organisms were identified in 19 cases, and $M$. malmoense and $M$. gordonae were identified in one case each.

Of the cultures identified as $M$. tuberculosis, 81 ( $98 \%$ ) were detected by LJ culture and $75(90 \%)$ by MB Check. MAC organisms were isolated in 16 of 19 and 15 of 19 cases by LJ and MB Check culture respectively. The two other atypical mycobacteria were isolated with the Bactec system only (table I).

Mycobacteria were demonstrated in 52 cultures $(13.5 \%)$ of the 386 selected specimens that were cultured with the Bactec system. Among these selected samples, $M$. tuberculosis was again seen most frequently ( 42 isolates), with 41 (98\%) detected by LJ culture, $38(91 \%)$ by MB Check and $19(45 \%)$ by Bactec. For the 10 atypical mycobacteria detected, the corresponding figures were six, five and eight cultures

Table I. Primary isolation of mycobacteria from 985 clinical samples by the MB Check biphasic culture system, culture on Löwenstein-Jensen solid egg medium and Bactec radiometry

\begin{tabular}{l|rccc}
\hline \multirow{2}{*}{ Species } & \multicolumn{4}{|c}{ Number of isolates } \\
\cline { 2 - 5 } & Total & MB Check & LJ culture & Bactec* \\
\hline$M$. tuberculosis & 83 & 75 & 81 & $19 / 42$ \\
$M$. avium complex & 19 & 15 & 16 & $6 / 8$ \\
$M$. malmoense & 1 & - & - & $1 / 1$ \\
$M$. gordonae & 1 & - & - & $1 / 1$ \\
Total & 104 & 90 & 97 & $27 / 52$ \\
\hline
\end{tabular}

* Bactec cultures were performed in $386(39 \%)$ of 985 cases; results are given as positive Bactec culture/total positive cultures of the 386 clinical samples.
Table II. Time for detection of $M$. tuberculosis and M. avium complex isolates with MB Check, LJ culture and Bactec systems

\begin{tabular}{lcll}
\hline \multicolumn{4}{c}{ Median (range) detection time (days)* } \\
\hline \multicolumn{1}{c}{ Species } & MB Check & LJ culture & Bactec \\
\hline M. tuberculosis & $19(4-53)$ & $20 \cdot 5(10-53)$ & $23(5-49)$ \\
M. avium complex & $17(6-50)$ & $23 \cdot 5(6-41)$ & $7 \cdot 5(6-35)$ \\
\hline
\end{tabular}

positive with the LJ, MB Check and Bactec systems respectively.

The detection time for $M$. tuberculosis was similar for all three culture systems; median time for detection was 19 (4-53) days for MB Check, 23 (5-49) days for Bactec, and $20.5(10-53)$ days for culture on $\mathrm{LJ}$ medium. The most rapid detection of MAC was achieved with the Bactec system (table II).

In 21 of the 90 positive MB Check cultures $(17 \mathrm{M}$. tuberculosis and four MAC), growth was detected first in the broth and around a week later on one or more of the solid agar slides.

The lowest contamination rate was seen for the MB Check system with $22(2.2 \%)$ of 985 , compared to 20 $(5.2 \%)$ of 386 for Bactec and $76(7.7 \%)$ of 985 for the solid LJ culture.

\section{Discussion}

Clinical diagnostic mycobacteriology is undergoing a period of rapid development. The potential importance of nucleic acid hybridisation techniques for the demonstration of mycobacteria in clinical specimens, especially after amplification of the target nucleic acid with polymerase chain reaction techniques, is increasingly recognised. Nevertheless, there is still a need for culture and isolation of mycobacteria, and for improvements in culture techniques, not least for the isolation of atypical mycobacteria. We have shown previously that conventional solid culture on LJ medium is not the optimal method for primary isolation of the clinically most important atypical mycobacteria. ${ }^{1,2}$ Broth culture as a complement to solid culture was introduced into mycobacterial laboratories with the Bactec system. Rapid and efficient primary isolation with the Bactec system has been reported..$^{1,2,8,8}$

A biphasic culture system, the MB Check, was developed by Giger and co-workers. They also reported the first evaluation of the system, based on the detection of $17 \mathrm{M}$. tuberculosis and 12 atypical mycobacteria. ${ }^{6}$ Our study confirms their findings of more rapid detection of mycobacterial growth with the MB Check system than with solid culture, accompanied with low contamination rates.

The relatively low sensitivity for detection of $M$. tuberculosis with the Bactec system, as well as its high sensitivity for isolation of MAC, in this study is in 
accordance with our earlier experience ${ }^{1}$ and also with the results reported by Salfinger. ${ }^{10}$ The poor results in demonstrating $M$. tuberculosis in both of these studies could be explained by the use of the sodium lauryl sulphate method for decontamination of samples. This method is not recommended for use with Bactec culture. The ratio of culture broth to inoculated sample material could also be important-it is much higher in the MB Check method, which allows toxic products in the inoculum to be more diluted than in Bactec cultures. ${ }^{10}$

Fourteen strains of mycobacteria (eight of $M$. tuberculosis, four of MAC, one of M. malmoense and one of M. gordonae) isolated with $\mathrm{LJ}$ or Bactec culture failed to grow in the MB Check system. After primary isolation, these strains were subcultured once on $\mathrm{LJ}$ medium and inoculated in the MB Check system. Good growth was seen in all cases, demonstrating that these isolates were capable of growing in the MB Check system. It is noteworthy that all strains detected with $\mathrm{LJ}$ culture and not with MB Check produced only a few colonies at primary isolation, suggesting the presence of small numbers of viable bacteria in these

\section{References}

1. Hoffner SE. Improved detection of Mycobacterium avium complex with the Bactec radiometric system. Diagn Microbiol Infect Dis 1988; 10: 1-6.

2. Hoffner SE, Henriques B, Petrini B, Källenius G. Mycobacterium malmoense: an easily missed pathogen. $J$ Clin Microbiol 1991 ; 29: 2673-2674.

3. Martin T, Cheke D, Natyshak I. Broth culture: the modern "guinea-pig" for isolation of mycobacteria. Tubercle 1989; 70: $53-56$.

4. Groothuis DG, Yates MD (eds). Manual of diagnostic and public health mycobacteriology, 2nd edn. London, European Society for Mycobacteriology. 1991.

5. Middlebrook G, Reggiardo Z, Tigertt WD. Automatable radiometric detection of growth of Mycobacterium tuberculosis in selective media. Am Rev Respir Dis 1977;115: 1066-1069. samples. Splitting of clinical samples with low mycobacterial numbers inherently leads to the risk of a nonhomogeneous distribution between the subsamples.

As is usually found when different methods for primary isolation are compared, no single method detected all positive samples. Conventional solid culture on LJ medium identified the most $(93 \%)$, followed by MB Check $(87 \%)$. This may indicate that eggbased solid culture medium is superior for isolating some mycobacterial strains. It is interesting that an egg-based solid medium is included in a recent modified version of the MB Check, introduced after this evaluation.

In conclusion, the MB Check system, with a combination of broth and solid mycobacterial culture, is easy to use and does not demand expensive equipment or radiolabelled broth. It provides rapid detection of $M$. tuberculosis and other clinically relevant mycobacteria with a sensitivity similar to that seen with $\mathrm{LJ}$ medium.

This study was supported by a grant from F. Hoffman-La Roche, Basel, Switzerland.

6. Giger T, Burkardt HJ. Evaluation of a new biphasic culture system for the recovery of mycobacteria. Eur J Clin Microbiol Infect Dis 1990; 9: 428-431.

7. Kent PT, Kubica GP (eds). Public health mycobacteriology, a guide for the level III laboratory. Atlanta, Centers for Disease Control. 1985

8. Fadda G, Roe SL. Recovery and susceptibility testing of Mycobacterium tuberculosis from extrapulmonary specimens by the Bactec radiometric method. J Clin Microbiol $1984 ; 19: 720-721$.

9. Damato JJ, Collins MT, Rothlauf MV, McClatchy JK. Detection of mycobacteria by radiometric and standard plate procedures. J Clin Microbiol 1983; 17 : 1066-1073.

10. Salfinger M, Demchuk BS, Kafader FM. Comparison between $M B$ check broth radiometric and conventional methods for recovery of mycobacteria from respiratory specimens. $J$ Microbiol Methods 1990; 12: 97-100. 